of $82 \mathrm{mmHg}$ ] and diastolic [to a minimum of $50 \mathrm{mmHg}$ ] BP, respectively; $n=604$ ) or having incident estimated glomerular filtration rate below $60 \mathrm{ml} / \mathrm{min} / 1.73 \mathrm{~m}^{2}\left(P_{\text {trend }}=0.03\right.$ and $P_{\text {trend }}=0.001$, respectively; $n=547$ ). The effects were strongest in participants with systolic BP $<120 \mathrm{mmHg}$ or diastolic BP $<70 \mathrm{mmHg}$, and were independent of glycemic control and other potential confounders. In the 140 subjects with proteinuria at baseline, lower systolic or diastolic BP was associated with a greater likelihood of proteinuria regression during follow-up.

Low BP-even below the currently accepted normal range - could, therefore, be protective against chronic kidney disease over the long term in patients with type 1 diabetes. The authors state, however, that randomized, controlled intervention trials would be needed to confirm whether actively lowering BP to below the current normal range prevents kidney disease in these individuals.

Original article Shankar A et al. (2007) Relationship between low-normal blood pressure and kidney disease in type 1 diabetes. Hypertension 49: 48-54

\section{Spironolactone add-on therapy decreases proteinuria in diabetic nephropathy}

Drugs that interfere with the renin-angiotensin system can provide renoprotection in patients with diabetic nephropathy, possibly by exerting an antiproteinuric effect. Aldosterone might have a role in the progression of renal disease; therefore, van den Meiracker et al. investigated whether adding the aldosterone receptor antagonist spironolactone to renin-angiotensin system inhibition improved proteinuria in patients with diabetic nephropathy.

Patients with type 2 diabetes and macroalbuminuria despite use of an angiotensin-converting-enzyme inhibitor or an angiotensin-receptor blocker for $\geq 1$ year were randomized to spironolactone $(n=29)$ or placebo $(n=30) 25-50 \mathrm{mg}$ daily for 1 year. Five patients taking spironolactone and one patient on placebo developed hyperkalemia within 2-12 weeks of initiation of study medication and were excluded.

After 1 year, urinary protein:creatinine and urinary albumin:creatinine ratios decreased markedly in patients treated with spironolactone but did not change in those receiving placebo.
Decreases in systolic and diastolic blood pressures were seen in patients treated with spironolactone, but no changes were noted in placebo-treated patients. Estimated glomerular filtration rate decreased in both groups, but the average decrease after 1 year was greater in the spironolactone group $(P=0.004)$. Increases in serum potassium levels, however, were seen in spironolactone-treated but not placebo patients. Urinary protein:creatinine and urinary albumin:creatinine ratios decreased with decreasing estimated glomerular filtration rate.

The authors conclude that adding spironolactone to an angiotensin-converting-enzyme inhibitor or an angiotensin-receptor blocker reduces proteinuria throughout 1 year of treatment; they add that the initial daily dose should be $\leq 25 \mathrm{mg}$ to reduce the risk of hyperkalemia.

Original article van den Meiracker AH et al. (2006) Spironolactone in type 2 diabetic nephropathy: effects on proteinuria, blood pressure and renal function. J Hypertens 24: $2285-2292$

\section{Rosiglitazone can be used for diabetes control in hemodialysis patients with hepatitis}

Administration of thiazolidinediones such as rosiglitazone could aid the control of diabetes in patients on maintenance hemodialysisincluding those with hepatitis B or C infection-claims a recent study. These agents target insulin resistance, which is prevalent in patients on long-term dialysis and is associated with type 2 diabetes and cardiovascular disease.

Rosiglitazone was administered to 78 hemodialysis patients, 25 of whom had hepatitis $B$ or C infection. Over a mean 15.4 months of treatment, rosiglitazone (up to $8 \mathrm{mg} /$ day) resulted in improved control of diabetes, with a response rate (glycated hemoglobin $<7 \%$ ) of $86.1 \%$. The agent markedly reduced triglyceride levels, and increased adiponectin levels (possibly as a result of insulin sensitization). Offsetting this benefit, however, was a significant increase in body weight $(P<0.01)$ as well as a possible negative impact on cardiovascular reserve (increased cardiothoracic ratio). Liver aminotransferase levels did not increase significantly in patients with viral hepatitis infection compared with those without, leading the authors to conclude that rosiglitazone is safe in hepatitis-infected patients on dialysis. 